\title{
NEUROPSICOLOGÍA Y EDUCACIÓN: CREATIVIDAD, INTELIGENCIAS MÚLTIPLES Y RENDIMIENTO ACADÉMICO EN EDUCACIÓN PRIMARIA
}

\author{
Neuropsychology and education: Creativity, multiple \\ intelligences and academic performance in primary \\ education
}

José Joaquín CaLdera OrTiz***, Fátima Llamas-SALGUERO*,

y Verónica LÓPEZ-FERNÁNDEZ*****

* Universidad Internacional de la Rioja

Correo-e:fatima.llamas@unir.net

* CEIP Rufino Blanco

Correo-e: jjcaldera30@gmail.com

**** Universidad Internacional de la Rioja

Correo-e:veronica.lopez@unir.net

Recibido: 19/06/2018; Aceptado: 28/11/2018; Publicado: 30/12/2018

Ref. Bibl. JOSÉ JOAQUÍN CALDERA ORTIZ, FÁTIMA LLAMAS-SALGUERO y VERÓNICA LÓPEZ-FERNÁNDEZ. Neuropsicología y educación: creatividad, inteligencias múltiples y rendimiento académico en Educación primaria. Enseñanza E Teaching, 36, 2-2018, 123-143.

RESUMEN: La creatividad y las inteligencias múltiples, variables neuropsicológicas relacionadas con la educación, son los pilares fundamentales para trabajar en las aulas de hoy en día. La formación de docentes, las nuevas estrategias, herramientas y aplicaciones metodológicas son esenciales y fundamentales para la generación de conocimiento en el alumno/a. El presente estudio persigue como objetivo principal analizar las relaciones existentes entre las variables neuropsicológicas: inteligencias múltiples, rendimiento académico y creatividad. Para llevar a cabo el estudio se ha seleccionado una muestra de 66 alumnos de la etapa de Educación Primaria 
JOSÉ JOAQUÍN CALDERA ORTIZ, FÁTIMA LLAMAS-SALGUERO Y VERÓNICA LÓPEZ-FERNÁNDEZ NEUROPSICOLOGÍA Y EDUCACIÓN: CREATIVIDAD, INTELIGENCIAS MÚLTIPLES Y RENDIMIENTO ACADÉMICO EN EDUCACIÓN PRIMARIA

del colegio público Rufino Blanco, de la localidad de Encinasola (Huelva). Se ha medido el nivel de inteligencias múltiples de cada alumno a través del Cuestionario de Detección de las Inteligencias Múltiples para alumnos de Primaria de McKenzie (1999) y la creatividad a través de la Prueba de Inteligencia Creativa CREA (Corbalán et al., 2003). Los datos necesarios para completar la variable rendimiento académico se han obtenido a través del sistema de gestión administrativa Séneca. El análisis de la información recogida en esta investigación se ha llevado a cabo desde una doble perspectiva: descriptiva, en un primer momento se clasificarán los datos obtenidos para cada una de las variables que son objeto de estudio, y correlacional, puesto que el siguiente paso fue realizar correlaciones entre las distintas variables. Los resultados obtenidos muestran la existencia de correlaciones positivas y estadísticamente significativas entre rendimiento académico e inteligencias múltiples y correlaciones no estadísticamente significativas entre creatividad y rendimiento académico y creatividad e inteligencias múltiples.

Palabras clave: creatividad; inteligencias múltiples; rendimiento académico; sistema escolar; neuropsicología.

SUMMARY: Creativity and multiple intelligences, neuropsychological variables, related to education, are the fundamental pillars to work in today's classrooms. Teacher training, new strategies, tools and methodological applications are essential and fundamental for the generation of knowledge in the student. The main objective of this study is to analyze the relationships between neuropsychological variables: multiple intelligences, academic performance and creativity. To carry out the study, a sample of 66 students from the primary education stage of the Rufino Blanco public school in the town of Encinasola (Huelva) was selected. The level of multiple intelligences of each student has been measured through the Multiple Intelligence Detection Questionnaire for McKenzie Elementary students (1999) and creativity through the CREA Creative Intelligence Test (Corbalán et al., 2003). The data needed to complete the variable academic performance have been obtained through the Seneca administrative management system. The analysis of the information gathered in this research has been carried out from a double perspective: descriptive, at first the data obtained for each one of the variables that are object of study and correlational will be classified, since the next step was correlations between the different variables. The results obtained show the existence of positive and statistically significant correlations between academic performance and multiple intelligences and non-statistically significant correlations between creativity and academic achievement and creativity and multiple intelligences.

Key words: creativity; multiple intelligences; academic achievement; school system; neuropsychology. 
JOSÉ JOAQUÍN CALDERA ORTIZ, FÁTIMA LLAMAS-SALGUERO Y VERÓNICA LÓPEZ-FERNÁNDEZ NEUROPSICOLOGÍA Y EDUCACIÓN: CREATIVIDAD, INTELIGENCIAS MÚLTIPLES Y RENDIMIENTO ACADÉMICO EN EDUCACIÓN PRIMARIA

\section{INTRODUCCIÓN}

La educación es uno de los factores que más influye en el avance y progreso de personas y sociedades. Además de proveer conocimientos, enriquece la cultura, el espíritu, los valores y todo aquello que nos caracteriza como seres humanos (Organización para la Cooperación y el Desarrollo Económico, 2016). Es necesaria en todos los sentidos y se ha considerado como un factor clave en el desarrollo de las personas. Sin embargo, las estadísticas y los rankings internacionales actuales no dejan en buen lugar a la educación en España (PISA, 2015). La falta de consenso en las políticas educativas y los actuales recortes en recursos económicos y materiales para implementar una educación de calidad son circunstancias que contribuyen a potenciar estos resultados.

En este escenario, es imprescindible hacer una reflexión sobre el conocimiento científico actual, relacionado con las capacidades del cerebro y la mente humana, para aprender y adquirir conocimientos. Este proceso debe ayudar a potenciar lo bueno que ya se tiene y a evitar caminos equivocados o infructuosos que pudieran contribuir a empeorar la situación (Morgado, 2012). La neurociencia y la educación deberían establecer, entre sí, una colaboración más estrecha. Las iniciativas que ya se han llevado a cabo en este sentido son importantes, pero insuficientes. La escuela no solo debería aprovechar los descubrimientos producidos en el ámbito de la neurociencia, sino que también debería demandar a los neurocientíficos la aclaración de ciertos temas de interés educativo (Marina, 2012). Entre esos temas, se encuentran aquellos que son objeto de estudio dentro de esta investigación: el rendimiento académico, las inteligencias múltiples o el desarrollo de técnicas creativas en el alumnado.

El rendimiento académico es una valoración externa del aprendizaje, y, en la medida en que este concepto esté construido de manera correcta, abarcará tanto el resultado como el proceso. Además, permite valorar el éxito o fracaso del sistema educativo. Tradicionalmente, se ha asociado el nivel de inteligencia a la capacidad y voluntad que tiene el alumno. Sin embargo, numerosas y valiosas investigaciones, como la teoría de las inteligencias múltiples de Gadner (1998), las investigaciones de Goleman (1999) o el modelo de mejora de competencias, han demostrado que el ser cognitivamente inteligente no implica un éxito a nivel personal y profesional. A pesar de todo esto, el rendimiento académico constituye una de las dimensiones más importantes dentro de los procesos de aprendizaje del alumnado.

A la hora de llevar a cabo una evaluación del rendimiento académico, para conocer, entre otros aspectos, como puede mejorarse, se analizan distintos factores que pueden influir en él. Entre estos factores, se encuentran: factores socioeconómicos, los programas de estudio, las metodologías de enseñanza utilizadas, la dificultad de emplear una enseñanza personalizada, los conceptos previos que tienen los alumnos, así como el nivel de pensamiento formal de los mismos (Benítez, Giménez y Osicka, 2000), sin embargo, Jiménez (2000) refiere que "se puede tener una buena capacidad intelectual y unas buenas aptitudes y sin embargo no estar obteniendo un rendimiento adecuado» (p. 21), ante la disyuntiva y con la 

Y RENDIMIENTO ACADÉMICO EN EDUCACIÓN PRIMARIA

perspectiva de que el rendimiento académico es un fenómeno multifactorial. Por ello, establecer una delimitación de rendimiento académico resulta bastante complicado (Abalde, Barca, Muñoz Ziemer, 2009).

Navarro (2003) utiliza el término constructo para referirse al rendimiento académico, haciendo referencia a que adopta valores cuantitativos y cualitativos que nos permiten conocer los conocimientos, habilidades, actitudes y valores que los discentes van desarrollando a lo largo del proceso de aprendizaje. Gómez-Castro (1996) lo define como el nivel que poseen los alumnos en conocimientos y destrezas escolares y que puede ser medido en cualquier proceso de evaluación. Diversas investigaciones educativas hacen referencia al concepto de rendimiento académico, concluyendo en una gran diversidad de líneas de estudio que permiten comprender su importancia dentro del acto educativo. Esta importancia es una de las razones que provoca que la sociedad española se encuentre bombardeada por informaciones que comparan el sistema educativo español con el resto de la Comunidad Europea, e incluso con otros sistemas de ámbito mundial.

El Informe PISA realizado por la OCDE en 2015 y publicado en la página web del Ministerio de Educación en 2016 sitúa al sistema educativo español en los últimos puestos, por debajo de la media de la OCDE y establecen la variable rendimiento académico como una de las más valoradas. El rendimiento de los alumnos españoles los sitúa cerca de países como Estados Unidos, Francia, Austria, Suecia, República Checa y Letonia, pero lejos de los primeros puestos ocupados por países como Singapur, Japón, Estonia o Finlandia.

Sin embargo, numerosos académicos han criticado a PISA por tratarse de una prueba que solo valora la calidad de los sistemas educativos en función de valores cuantitativos y, en su opinión, no sirve para valorar la calidad real de la educación de un país. Tradicionalmente, el rendimiento académico se ha asociado al nivel de inteligencia y a la capacidad de voluntad que tiene el alumno. Sin embargo, con el tiempo se han llevado a cabo diferentes estudios que demuestran que en el rendimiento influyen muchos más factores (Rodríguez, 1982). Goleman (1999) establece que alumnos con bajo rendimiento escolar también presentan deficiencias en su inteligencia emocional. En segundo lugar, Campos y González (1993) establecen que la creatividad influye en el rendimiento académico. Por ello, pasamos en los siguientes párrafos a analizar de forma más detallada estos dos factores.

Gadner $(1983,1998)$ indica que no existe una única inteligencia, defendiendo una visión plural de la misma. Esta visión de la inteligencia es apoyada por otros psicólogos como Guilford (1967), Thursstone (1939) y especialmente Sternberg (1985). Por lo tanto, según su obra, hay muchas formas de ser inteligente, siendo al menos ocho las inteligencias que nos definen.

En un principio, Gardner parecía estar de acuerdo con lo postulado en la teoría cognitiva de Piaget y su idea de las cuatro etapas del desarrollo cognitivo: etapa sensoriomotora, preoperacional, de operaciones concretas y de operaciones formales; pero cuando comenzó sus propias investigaciones, durante los años ochenta, sus ideas sobre el aprendizaje cambiaron (Lazear, 1991b). Gardner había visto que 
JOSÉ JOAQUÍN CALDERA ORTIZ, FÁTIMA LLAMAS-SALGUERO Y VERÓNICA LÓPEZ-FERNÁNDEZ NEUROPSICOLOGÍA Y EDUCACIÓN: CREATIVIDAD, INTELIGENCIAS MÚLTIPLES Y RENDIMIENTO ACADÉMICO EN EDUCACIÓN PRIMARIA

en las teorías planteadas por Piaget se ignoraban aspectos tan importantes como la biología y la creatividad humana (Gardner, 1993a). Su objetivo estaba centrado en poder encontrar y probar una teoría más amplia de la inteligencia, en la que tuviesen cabida aspectos como los anteriores, explicando la creatividad y la gama de roles que estaban presentes en la sociedad. En palabras de Gardner:

All focus on a certain kind of logical or linguistic problem solving; all ignore biology; all fail to come to grips with the higher levels of creativity; and all are insensitive to the range of roles highlighted in human society (1993a: 24).

Adhering to the traditional notion of intelligence, schools identify certain skills as basic or essential, and they demean others by labeling them as frills. Narrowly defined limits of intelligent behavior make students who don't excel in linguistic or mathematical disciplines perceive their talents to be of little use (Campbell y Campbell, 1999: 7).

Gardner (2005) destaca su inspiración para encontrar una teoría más compasiva:

I believe this theory of intelligence may be more humane and more veridical than alternative views of intelligence and that it more adequately reflects the data of human «intelligent» behavior.

Gardner (2005) concluye que las personas son diferentes y que, por tanto, tienen diferentes capacidades de pensar y diversas maneras de aprender. Esta teoría pluralista demuestra que cada alumno es único y responde a esto mediante el desarrollo de la instrucción basada en las diferencias de los alumnos. Como señala Fonseca Mora (2007):

Este concepto de inteligencia o capacidades reconoce la diversidad, la existencia de distintas formas de ser que son de igual estatus. Ser una persona «inteligente» puede significar tener una gran capacidad memorística, tener un amplio conocimiento, pero también puede referirse a la capacidad de conseguir convencer a los demás, saber estar, expresar de forma adecuada sus ideas ya sea con las palabras o con cualquier otro medio de índole artística, controlar su ira, o saber localizar lo que se quiere, es decir, significa saber solucionar distintos problemas en distintos ámbitos. Además, la formación integral de los alumnos ha de entenderse también como la formación de lo emocional y no sólo como formación de lo cognitivo (p. 2).

Según Gardner (2005) una inteligencia es una habilidad que puede servir para resolver problemas o crear productos de necesidad. La inteligencia representa una colección de potencialidades biopsicológicas que van mejorando con la edad. Él considera que es mejor describir la competencia cognitiva humana usando el término inteligencias, que agrupa los talentos, habilidades y capacidades mentales de un individuo. Afirma que todo individuo tiene cada una de estas inteligencias, aunque una persona podría ser más talentosa en una inteligencia que en otras. También, varía en la combinación de inteligencias y la capacidad de desarrollarlas (Arnold y Fonseca, 2004; Gardner, 2005). Asimismo, Gardner (2006) dice que casi 
JOSÉ JOAQUÍN CALDERA ORTIZ, FÁTIMA LLAMAS-SALGUERO Y VERÓNICA LÓPEZ-FERNÁNDEZ NEUROPSICOLOGÍA Y EDUCACIÓN: CREATIVIDAD, INTELIGENCIAS MÚLTIPLES Y RENDIMIENTO ACADÉMICO EN EDUCACIÓN PRIMARIA

todos los roles culturales requieren una combinación de inteligencias. La mayoría de las personas funcionan con una o dos inteligencias que se encuentran muy desarrolladas, teniendo las otras en un estado de espera (Smith, 2001).

En su búsqueda de un concepto de la inteligencia diferente y más compasiva, Gardner (2006) tuvo que investigar la evidencia de varias fuentes y decidió utilizar ocho criterios para identificar las inteligencias y considerarlas parte de su teoría.

In coming up with the list, I reviewed evidence from various sources: knowledge about normal development and development in gifted individuals; information about the breakdown of cognitive skills under conditions of brain damage; studies of exceptional populations, including prodigies, savants, and autistic children; data about the evolution of cognition over the millennia; cross-cultural accounts of cognition; psychometric studies, including examinations of correlations among tests; and psychological training studies, particularly measures of transfer and generalization across tasks (p. 7).

Las distintas fuentes que Gardner empleó fueron:

- Estudio de regiones cerebrales dañadas.

- Existencia de sabios, niños prodigios, y otras personas excepcionales.

- Existencia de una o más funciones cerebrales que desempeñan una función esencial.

- Un grupo definible de acciones que indican el dominio de las habilidades.

- La verosimilitud a través de la evolución.

- Una susceptibilidad de la codificación de un sistema de símbolos.

- El apoyo de las tareas psicológicas que revelan que unas habilidades son (o no son) manifestaciones de las mismas inteligencias.

Después de considerar todos estos criterios, Gardner inicialmente identificó siete inteligencias, a las que después añadió una más y que a continuación se presentan junto a su localización cerebral (véase Tabla 1):

TABLA 1

Localización cerebral de las inteligencias múltiples

\begin{tabular}{|l|l|}
\hline \multicolumn{1}{|c|}{ INTELIGENCIA } & \multicolumn{1}{c|}{ LOCALIZACIÓN CEREBRAL } \\
\hline Inteligencia lingüística & Lóbulo temporal y frontal izquierdos \\
\hline Inteligencia lógico-matemática & Lóbulo parietal derecho y frontal izquierdo \\
\hline Inteligencia espacial & Hemisferio derecho \\
\hline Inteligencia corporal-kinestésica & Cerebelo, los ganglios basales y córtex motor \\
\hline Inteligencia musical & Lóbulo temporal derecho \\
\hline Inteligencia interpersonal & Lóbulo frontal \\
\hline Inteligencia intrapersonal & Lóbulo frontal \\
\hline Inteligencia naturalista & Hemisferio derecho \\
\hline
\end{tabular}



Y RENDIMIENTO ACADÉMICO EN EDUCACIÓN PRIMARIA

La teoría de Gardner ha supuesto una revolución en el ámbito de la psicología y de la educación pues su aplicación supone un giro en el sistema educativo que, tradicionalmente, ha dado protagonismo a las áreas lingüística y matemática. De esta manera, la educación se convierte en una educación personalizada e individualizada para cada alumno (Fernández y Mihura, 2015). La autonomía de las inteligencias, la interacción de las mismas y la posibilidad de desarrollar cada una es lo que ha hecho que tenga una aplicación directa en el campo educativo, porque ofrece un marco nuevo para ubicar a cada alumno de mejor manera en el proceso de aprendizaje (Aliaga et al., 2012). Analizar estas inteligencias en el alumnado, se convierte en un factor clave, debido a la gran diversidad de estudiantes que se encuentran en las aulas. Conocer y utilizar los puntos fuertes de cada estudiante permite no solo sacar lo mejor de cada uno, sino que, además, posibilita la utilización de estos puntos fuertes como herramientas para mejorar sus debilidades, utilizando metodologías basadas en tender puentes.

Como se señalaba anteriormente, Gardner observó que la creatividad era otro elemento fundamental que las teorías de Piaget no habían tenido en cuenta a la hora de describir la inteligencia. Sin embargo, consideró que la creatividad está intrínsecamente ligada al ser humano (Fuentes y Torbay, 2004). Es de vital importancia para tomar decisiones, para saber interpretar y acceder a la información, así como para desarrollar capacidades que permitan al ser humano tener una actitud positiva, coherente, creativa y crítica hacia la vida, desarrollando de esta forma la constancia, la autonomía, la curiosidad, la imaginación (Blázquez, 2009).

Uno de los principales exponentes del tema es Guilford (1991), quien a mediados del siglo xx propone el término de creatividad, y postula que esta y la inteligencia no son lo mismo, señalando que ambas son habilidades homólogas pero diferentes. Para este teórico, la "creatividad" es entendida como una forma distinta de inteligencia. Así, Guilford (1991) la denomina "pensamiento divergente» en contraposición al "pensamiento convergente» que, tradicionalmente, se medía en las pruebas (test) más comunes de inteligencia (Espíndola, 1996). Marcó la distinción entre el pensamiento convergente y el divergente. Desde ese momento, la creatividad se ha considerado como un elemento esencial en cualquier estudio formal referido al intelecto humano. A lo expuesto anteriormente, también se le puede unir el concepto de imaginación, para apoyar la definición de creatividad. Según Ken Robinson, la creatividad se define a partir de tres conceptos claves como son: la imaginación, que es fuente de creatividad; el proceso de generar ideas originales, que tienen valor, y la innovación (Braun, 2012).

Recopilando lo expuesto con anterioridad, se extrae la conclusión de que las ideas creativas se originan gracias al conocimiento de las personas, aunque este, por sí solo, no puede determinar el grado de creatividad que presenta un individuo. Lo verdaderamente importante radica en la forma que tenga una persona de manejar ese conocimiento y en la actitud curiosa y positiva que esta desarrolle para el logro de este producto creativo (Blasco, Bueno, Navarro y Torregrosa, 2002). De acuerdo con Elisondo y Donolo (2011), la creatividad se ha definido a través 

Y RENDIMIENTO ACADÉMICO EN EDUCACIÓN PRIMARIA

del estudio de las características personales y de las del producto creativo. De este modo, estos autores exponen que, aunque existen múltiples definiciones, estas dos características y la relación que existe entre ambas, así como el contexto donde se desarrollan, deben tenerse en cuenta.

En la actualidad, nadie cuestiona la necesidad de desarrollar la creatividad, sin embargo, aún es insuficiente la preparación que tienen algunos profesores. Para conocer cómo funciona un proceso mental, en este caso la creatividad, es necesario indagar en los procesos cerebrales y las áreas que se ponen en marcha para desarrollar esta capacidad. De este modo, Sastre y Pascual (2013) exponen la importancia que se concede al acto creativo desde la neurociencia. Por otro lado, pocos currículos abordan estos problemas con especificidad y solidez, y pocos son, también, los cursos de postgrado que están encaminados al logro de una formación efectiva de los profesores para el logro de este empeño. Esto trae, como consecuencia, que algunos profesores, al desconocer las formas para evaluar la efectividad de sus estrategias metodológicas y carecer de un sistema de indicadores y técnicas para evaluar el desarrollo de la creatividad en sus estudiantes, desarrollan acciones didácticas que no se corresponden con la verdadera complejidad de la creatividad y del proceso de su desarrollo, las cuales no aportan los resultados esperados.

Es necesario el desarrollo del pensamiento creativo en el ámbito escolar. Autores como M. Romo (2012) afirman que en las escuelas, actualmente, se separa la creatividad de la educación. La escuela, cuando incluye la creatividad, lo hace como algo al margen e incide sobre todo en el concepto de original, nuevo o diferente olvidando que creatividad también implica utilidad, validez y pensamiento crítico. Calero (2011) insiste en la importancia de motivar el pensamiento creativo desde una edad temprana, en parte, porque las características propias de esta edad evolutiva hacen que sea un periodo muy propicio para ello: la motivación por la experimentación, la curiosidad por descubrir y conocer cosas nuevas, etc. Pero este fomento de la creatividad debe ser completado desde el ámbito familiar. Desde estos dos contextos, se pueden desarrollar actividades que fomenten el pensamiento creativo. La clave, según exponen López (2005) y Calero (2011), radica en proporcionar el ambiente adecuado para el desarrollo de esta capacidad.

Por tanto, y teniendo en cuenta la literatura relacionada con esta temática, se ha considerado adecuado desarrollar la presente investigación. El objetivo principal que se persigue con la misma es analizar la relación existente entre el rendimiento académico, la creatividad y las inteligencias múltiples. Se espera encontrar relaciones positivas y estadísticamente significativas. Existen diferentes investigaciones que muestran resultados positivos entre estas variables. En lo relativo a la relación entre rendimiento académico e inteligencias múltiples, la mayoría de las investigaciones apuntan a la inteligencia como un gran predictor del rendimiento académico (Cascón, 2000; Elshout y Veenman, 1992; Jensen, 1998 y Neisser et al., 1996). De la misma forma observamos investigaciones cuyos resultados demuestran una relación positiva entre creatividad y rendimiento académico, llegando a considerar a 
JOSÉ JOAQUÍN CALDERA ORTIZ, FÁTIMA LLAMAS-SALGUERO Y VERÓNICA LÓPEZ-FERNÁNDEZ NEUROPSICOLOGÍA Y EDUCACIÓN: CREATIVIDAD, INTELIGENCIAS MÚLTIPLES

Y RENDIMIENTO ACADÉMICO EN EDUCACIÓN PRIMARIA

la primera como una variable predictora de la segunda (Campos y González, 1993; Escalante, 2005; Moreno, 1992; Ullmann, 1972). Por último, encontramos estudios que ponen de manifiesto una relación positiva entre la inteligencia y la creatividad (Mednick, 1962; Renzulli, 1977; Sternberg, 1988; Weisberg y Alba, 1981; Weisberg, 1988).

\section{Metodología}

El objetivo principal que se persigue es analizar la relación existente entre el rendimiento académico, la creatividad y las inteligencias múltiples. Para conseguir este objetivo general, se plantean los siguientes objetivos específicos: identificar el grado de creatividad e inteligencias múltiples en alumnos de educación primaria, determinar si las inteligencias múltiples tienen relación con la creatividad, analizar si la creatividad está relacionada con el rendimiento académico y determinar si el rendimiento académico está relacionado con las inteligencias múltiples. Atendiendo a los resultados obtenidos se espera que:

- Hipótesis 1: Existan correlaciones positivas y estadísticamente significativas entre el rendimiento académico y las inteligencias múltiples.

- Hipótesis 2: Aparezcan correlaciones positivas y estadísticamente significativas entre las inteligencias múltiples y la creatividad.

- Hipótesis 3: Existan correlaciones positivas y estadísticamente significativas entre el rendimiento académico y la creatividad.

\subsection{Diseño}

El análisis de la información recogida en esta investigación se ha llevado a cabo desde una doble perspectiva: descriptiva, puesto que en un primer momento se clasificaron los datos obtenidos para cada una de las variables que son objeto de estudio y correlacional, puesto que el siguiente paso fue realizar correlaciones entre las distintas variables. Se ha llevado a cabo un estudio ex post facto puesto que en primer lugar se produjeron los hechos y después se analizaron las causas y consecuencias (Bernardo y Calero, 2000); correlacional, puesto que se ha pretendido conocer y evaluar la relación que existe entre las distintas variables (Hernández, 2003) y transversal, ya que se midió en una población definida y en punto específico de tiempo, en el que se realizó una investigación empirista positiva de carácter cuantitativo (Martínez, 2007).

\subsection{Población y muestra}

La muestra seleccionada la componen un total de 66 alumnos de Educación Primaria en el CEIP Rufino Blanco (Encinasola). Esta muestra representa la totalidad 

NEUROPSICOLOGÍA Y EDUCACIÓN: CREATIVIDAD, INTELIGENCIAS MÚLTIPLES Y RENDIMIENTO ACADÉMICO EN EDUCACIÓN PRIMARIA

de los alumnos que cursan la etapa de Educación Primaria. Se trata de una muestra seleccionada por conveniencia, donde los sujetos participantes han sido elegidos debido a la proximidad y accesibilidad para el investigador.

Desde hace 3 años, es un centro reconocido por la administración educativa como Comunidad de Aprendizaje y, actualmente, se encuentra inmerso en el desarrollo de un proyecto de investigación aprobado por la Consejería de Educación de la Junta de Andalucía relativo a la Neuropsicología y educación. Esta investigación ha supuesto el primer paso a la hora de poner en marcha este proyecto. El rango de edades de los componentes de la muestra es de 5 a 13 años $(M=8,48 ; D T=1,81)$. Respecto al sexo, la muestra está compuesta por un $37,87 \%$ de chicas y un $62,12 \%$ de chicos. Si hacemos una diferenciación por cursos, se observa que 8 alumnos son de $1 .^{\circ}$ de primaria ( 3 chicas y 5 chicos), 8 alumnos de $2 .^{\circ}$ de primaria $(3$ chicas y 5 chicos), 16 alumnos de $3 .^{\circ}$ de primaria ( 5 chicas y 11 chicos), 13 alumnos de $4 .^{\circ}$ de primaria ( 5 chicas y 8 chicos), 13 alumnos de $5 .^{\circ}$ de primaria ( 6 chicas y 7 chicos) y 7 alumnos de $6 .^{\circ}$ de primaria ( 3 chicas y 4 chicos).

\subsection{Variables medidas e instrumentos aplicados}

Para llevar a cabo este estudio se han tenido en cuenta tres variables: rendimiento académico, inteligencias múltiples y creatividad. Se procede, a continuación, a describir cada una ellas incluyendo los instrumentos que se han empleado para realizar su medición.

La variable rendimiento académico ha sido medida obteniendo el resultado derivado del cálculo de la media de todas las calificaciones finales obtenidas por cada alumno en las diferentes asignaturas durante el segundo trimestre del curso escolar 2015/2016. La información ha sido proporcionada por la dirección del centro, utilizando para ello el sistema de gestión administrativa Séneca.

Para realizar la medición de las inteligencias múltiples se ha utilizado el Cuestionario para el alumno de Infantil y Primaria de Inteligencias Múltiples adaptado por McKenzie (1999) para población española, con una muestra de 66 alumnos de educación primaria (Ejemplar para el alumno de Primaria. Adaptación de Walter McKenzie, 1999). El cuestionario tiene ocho apartados dedicados a cada una de las inteligencias propuestas por Gardner. Cada uno de ellos está compuesto por diez frases que describen situaciones relacionadas con la inteligencia en concreto y el sujeto debe contestar, en el lugar correspondiente, con la siguiente puntuación: (1) si se siente identificado con la frase, $(0,5)$ si la situación lo representa algunas veces y (0) en el caso de no identificarse nunca.

En último lugar, para medir la variable creatividad se ha empleado la prueba CREA. Inteligencia Creativa. Una medida cognitiva de la creatividad (CREA; Corbalán et al., 2003). La aplicación de esta prueba permite conocer el nivel de creatividad que tiene una persona. Para valorar el pensamiento divergente emplea una escala tipo Likert de tres puntos: 1 = baja, 2 = media y $3=$ alta. Dicho test contiene tres subpruebas (CREA A, CREA B y CREA C), de forma que se utilizan unas u otras en 
JOSÉ JOAQUÍN CALDERA ORTIZ, FÁTIMA LLAMAS-SALGUERO Y VERÓNICA LÓPEZ-FERNÁNDEZ NEUROPSICOLOGÍA Y EDUCACIÓN: CREATIVIDAD, INTELIGENCIAS MÚLTIPLES Y RENDIMIENTO ACADÉMICO EN EDUCACIÓN PRIMARIA

función de la edad que presenten los sujetos que componen la muestra. En la presente investigación se ha utilizado la subprueba CREA C, ya que la muestra tiene una media de 8,48 años.

Dicha prueba consiste en la presentación de una imagen en blanco y negro a los alumnos, a partir de la cual y en un periodo de 4 minutos, previa instrucción del administrador de la prueba, tienen que escribir todas las preguntas que les inspire esa imagen. Posteriormente, dichas preguntas se analizan por el investigador y se aplica una fórmula matemática que permite conocer su puntuación creativa. Para conocer en qué grupo de creatividad se encuentra el sujeto (baja, media, alta), es necesario identificar la puntuación centil. Esta puntuación se obtiene al comparar la puntuación obtenida con el centil que le correspondería en una tabla que aporta el manual, adaptada a población española y ampliamente validada.

\subsection{Procedimiento}

En un primer momento se procede a plantear el problema de investigación. Esta inquietud surge por el deseo de poner en marcha en este centro nuevas metodologías, basadas en la investigación y la innovación educativa, que permitan mejorar los resultados y el rendimiento académico del alumnado. Posteriormente, se realiza una revisión bibliográfica exhaustiva sobre cada una de las variables objeto de estudio, así como de sus bases neuropsicológicas. Todo ello permite contextualizar la investigación y establecer un marco teórico.

Una vez sentadas las bases teóricas, se procedió a realizar la selección del centro donde se llevaría a cabo el estudio. Esta selección estuvo determinada principalmente porque el investigador desempeña su labor docente en el mismo, facilitando de esta forma distintos aspectos del proceso, como, por ejemplo, el acceso a la información o la recogida de datos. A continuación, se solicita una reunión con el equipo directivo para comunicarle los aspectos principales del estudio y de la misma surge la idea de organizar dos reuniones. La primera se realizó con todos los profesores que imparten docencia en la etapa de primaria y tuvo como principal objetivo informarles sobre el estudio y su desarrollo, así como para solicitarles su colaboración en el desarrollo de las pruebas, y la segunda se realizó con los padres a los cuales, además de explicarles el objetivo de la investigación, se les pasó un consentimiento informado ad hoc que posteriormente entregaron a la dirección del centro.

El siguiente paso que se llevó a cabo fue la administración de los cuestionarios en las distintas aulas. La prueba de inteligencias múltiples fue pasada de forma colectiva y simultánea a todos los alumnos de cada nivel. Se realizó durante tres días consecutivos realizando dos cursos por día. El procedimiento que se siguió en todos los cursos consistió en la lectura en voz alta de las instrucciones de cada una de las pruebas, para instar posteriormente a los sujetos a que comenzaran con su desarrollo. Se realizó el Cuestionario de Inteligencias Múltiples de McKenzie (1999). 
JOSÉ JOAQUÍN CALDERA ORTIZ, FÁTIMA LLAMAS-SALGUERO Y VERÓNICA LÓPEZ-FERNÁNDEZ NEUROPSICOLOGÍA Y EDUCACIÓN: CREATIVIDAD, INTELIGENCIAS MÚLTIPLES Y RENDIMIENTO ACADÉMICO EN EDUCACIÓN PRIMARIA

Posteriormente, se pasó a realizar la evaluación de la prueba. Esta evaluación fue realizada por el investigador.

En segundo lugar, se les administró la subprueba del test CREA C (Corbalán et al., 2003). El procedimiento a seguir fue similar al anterior, pero llevado a cabo de manera individual, empleando un tiempo de cuatro minutos para cada uno de los sujetos. Se hizo en un aula habilitada al efecto durante tres días, realizando la prueba a 22 sujetos cada día, durante tres días consecutivos y posteriores a la realización de la prueba de inteligencias múltiples. La evaluación, al igual que en el caso anterior, fue realizada posteriormente por el investigador.

Finalmente, con el objetivo de conseguir los datos necesarios para completar la variable rendimiento académico, se solicitaron al equipo directivo los resultados académicos obtenidos por el alumnado de toda la etapa de primaria durante el segundo trimestre del curso 2015/16. Posteriormente, el investigador calculó la media obtenida por cada alumno teniendo en cuenta los resultados obtenidos en cada una de las materias.

En la siguiente fase se organizan y analizan los datos que permiten obtener los resultados de las pruebas y los indicadores estadísticos correspondientes. Y, por último, se establecen las conclusiones del estudio.

\subsection{Análisis de datos}

Para el análisis correlacional y puesto que las variables son cuantitativas, se utilizó el coeficiente de correlación de Pearson, realizado con el programa spss. Con el objetivo de analizar las relaciones existentes entre las diferentes variables, se tuvo en cuenta el valor de $p$, siendo los valores menores o iguales a ,05 los que indican que la relación establecida entre las variables es significativa.

\section{Resultados}

\subsection{Estadisticos descriptivos}

\subsubsection{Estadísticos descriptivos de las inteligencias múltiples}

Atendiendo a los resultados obtenidos en el Cuestionario de Detección de las Inteligencias Múltiples para el alumno de Infantil y Primaria (McKenzie, 1999), se observa que la mayoría de los alumnos obtuvieron valores medios para cada una de las inteligencias analizadas, siendo la puntuación media más alta la obtenida en la inteligencia corporal-kinestésica $(6,61)$ y la más baja la obtenida en las inteligencias naturalistas e intrapersonal $(6,04)$. Respecto a los descriptivos mediana y moda, la mayor parte de los valores se sitúan en una puntuación 6,00. Observamos que los valores mínimos obtenidos por la muestra difieren bastante respecto a los valores máximos. 
JOSÉ JOAQUÍN CALDERA ORTIZ, FÁTIMA LLAMAS-SALGUERO Y VERÓNICA LÓPEZ-FERNÁNDEZ

NEUROPSICOLOGÍA Y EDUCACIÓN: CREATIVIDAD, INTELIGENCIAS MÚLTIPLES

Y RENDIMIENTO ACADÉMICO EN EDUCACIÓN PRIMARIA

TABLA 2

Estadísticos descriptivos de los resultados obtenidos en la prueba de inteligencias múltiples

\begin{tabular}{|c|c|c|c|c|c|c|c|c|c|}
\hline & & I.L. & I.L.M. & I.E. & I.N. & I.C.K. & I.INTER. & I.M. & I.INTRA. \\
\hline $\mathrm{N}$ & Válidos & 66 & 66 & 66 & 66 & 66 & 66 & 66 & 66 \\
\hline & Perdidos & 0 & 0 & 0 & 0 & 0 & 0 & 0 & 0 \\
\hline & dia & 6,394 & 6,235 & 6,402 & 6,045 & 6,614 & 6,205 & 6,523 & 6,045 \\
\hline & diana & 6,000 & 6,000 & 6,000 & 5,750 & 7,000 & 6,000 & 6,000 & 6,000 \\
\hline & & 5,0 & 6,0 & 6,0 & 5,0 & 7,0 & 6,0 & 6,0 & 6,0 \\
\hline & sv. típ. & 1,4611 & 1,1741 & 1,2160 & 1,3860 & 1,2582 & 1,1733 & ,9862 & 1,5031 \\
\hline & imo & 4,0 & 3,0 & 3,5 & 4,0 & 4,0 & 4,0 & 4,0 & 2,0 \\
\hline & ximo & 10,0 & 9,5 & 10,0 & 9,0 & 9,0 & 9,0 & 9,5 & 9,0 \\
\hline & $\begin{array}{l}\text { enda. I.L.: } \\
\text { acial; I.N.: } \\
\text { ligencia Ir }\end{array}$ & $\begin{array}{l}\text { ligenc } \\
\text { ligenc }\end{array}$ & Lingüí & ; I.L. & Inteli & cia Ló & Matem & $\begin{array}{l}\text { I.E. } \\
\mathrm{ca} \text { I }\end{array}$ & $\begin{array}{l}\text { teligencia } \\
\text { ter: }\end{array}$ \\
\hline
\end{tabular}

\subsubsection{Estadísticos descriptivos de la creatividad}

Atendiendo a los resultados recogidos en la prueba CREA. Inteligencia Creativa. Una medida cognitiva de la creatividad (CREA; Corbalán et al., 2003), se extraen las siguientes conclusiones. La media de la muestra $(38,04)$ se corresponde con un valor medio de creatividad tal y como se establece en el manual de la misma. Se observa en la Tabla 3 que el valor máximo obtenido $(84,00)$ muestra una gran diferencia con el valor mínimo $(4,00)$.

TABLA 3

Estadísticos descriptivos de los resultados obtenidos en la prueba de creatividad

\begin{tabular}{|l|l|r|}
\hline \multirow{2}{*}{$N$} & Válidos & 66 \\
\cline { 2 - 3 } & Perdidos & 0 \\
\hline Media & 38,0455 \\
\hline Mediana & 37,5000 \\
\hline Moda & 29,00 \\
\hline Desv. típ. & 19,03716 \\
\hline Mínimo & 4,00 \\
\hline Máximo & 84,00 \\
\hline
\end{tabular}




\subsubsection{Estadísticos descriptivos del rendimiento académico}

Los resultados académicos analizados corresponden a la nota media obtenida por el alumnado en el conjunto de las materias de la etapa de Educación Primaria en el segundo trimestre del curso escolar 2015-2016. Los datos que se muestran en la Tabla 4 señalan que el valor medio se sitúa en un 6.64. Esta línea se mantiene marcada en los valores obtenidos en la mediana y la moda $(7,00)$. La desviación típica es de 1,38. El valor máximo se sitúa en 10, existiendo una gran diferencia con el valor mínimo $(3,00)$.

TABLA 4

Estadísticos descriptivos del rendimiento académico

\begin{tabular}{|l|l|r|}
\hline \multirow{2}{*}{ Media } & Válidos & 66 \\
\cline { 2 - 3 } & Perdidos & 0 \\
\hline Mediana & 6,64 \\
\hline Moda & 7,00 \\
\hline Desv. típ. & 7 \\
\hline Mínimo & 1,388 \\
\hline Máximo & 3 \\
\hline
\end{tabular}

\subsection{Correlaciones}

3.2.1. Correlaciones entre el rendimiento académico y las inteligencias múltiples

Los resultados que pueden observarse en la Tabla 5 muestran una correlación positiva alta y estadísticamente significativa entre rendimiento académico e inteligencia lingüística $(r=, 88 ; p=, 00)$. Resultados similares se obtienen para la correlación entre rendimiento académico y las inteligencias lógico-matemáticas $(r=, 78$; $p=, 00)$ y espacial $(r=, 83 ; p=, 00)$. Se observa una correlación positiva moderada y estadísticamente significativa entre rendimiento académico y las inteligencias naturalista $(r=, 54 ; p=, 00)$, interpersonal $(r=, 56 ; p=, 00)$, musical $(r=, 65 ; p=$ $00)$ e intrapersonal $(r=, 48 ; p=, 00)$. Por último, se observa una correlación positiva baja y estadísticamente significativa entre rendimiento académico e inteligencia Corporal-Kinésica $(r=, 29 ; p=, 01)$. 
JOSÉ JOAQUÍN CALDERA ORTIZ, FÁTIMA LLAMAS-SALGUERO Y VERÓNICA LÓPEZ-FERNÁNDEZ NEUROPSICOLOGÍA Y EDUCACIÓN: CREATIVIDAD, INTELIGENCIAS MÚLTIPLES Y RENDIMIENTO ACADÉMICO EN EDUCACIÓN PRIMARIA

TABLA 5

Correlación entre inteligencias múltiples y el rendimiento académico

\begin{tabular}{|c|c|c|}
\hline INTELIGENCIA & CORRELACIÓN DE PEARSON & VALOR P \\
\hline I.L. &, 88 &, $00^{*}$ \\
\hline I.L.M. &, 78 &, $00^{*}$ \\
\hline I.E. &, 83 &, $00^{*}$ \\
\hline I.N. &, 54 &, $00^{*}$ \\
\hline I.C.K. &, 29 &, $01^{*}$ \\
\hline I.Inter. &, 56 &, $00^{*}$ \\
\hline I.M. &, 65 &, $00^{*}$ \\
\hline I.Intra. &, 48 &, $00^{*}$ \\
\hline $\begin{array}{l}\text { Leyenda. I.L.: Inteligencia Lingüística; I.L.M.: Inteligencia Lógico Matemática; I.E.: Inteligencia } \\
\text { Espacial; I.N.: Inteligencia Naturalista; I.C.K.: Inteligencia Corporal-Kinestésica; I.Inter: } \\
\text { Inteligencia Interpersonal; I.M.: Inteligencia Musical; I.Intra.: Inteligencia Intrapersonal. }\end{array}$ \\
\hline
\end{tabular}

\subsubsection{Correlaciones entre inteligencias múltiples y creatividad}

Los resultados que se presentan en la Tabla 6 muestran correlaciones negativas y no estadísticamente significativas entre inteligencia lingüística y creatividad $(r=$ -, 13; $p=27)$, inteligencia lógico matemática y creatividad ( $r=-, 13 ; p=26)$, inteligencia espacial y creatividad $(r=-, 14 ; p=23)$, inteligencia corporal-kinestésica y creatividad $(r=-, 17 ; p=89)$ e inteligencia musical y creatividad $(r=-, 12 ; p=33)$.

Por otra parte se obtienen correlaciones positivas y no estadísticamente significativas entre inteligencia naturalista y creatividad $(r=, 05 ; p=65)$, inteligencia interpersonal y creatividad $(r=, 12 ; p=33)$ e inteligencia intrapersonal y creatividad $(r=, 02 ; p=84)$. 
JOSÉ JOAQUÍN CALDERA ORTIZ, FÁTIMA LLAMAS-SALGUERO Y VERÓNICA LÓPEZ-FERNÁNDEZ NEUROPSICOLOGÍA Y EDUCACIÓN: CREATIVIDAD, INTELIGENCIAS MÚLTIPLES Y RENDIMIENTO ACADÉMICO EN EDUCACIÓN PRIMARIA

TABLA 6

Correlación entre inteligencias múltiples y creatividad

\begin{tabular}{|c|c|c|}
\hline INTELIGENCIA & CORRELACIÓN DE PEARSON & VALOR P \\
\hline I.L. &,- 13 &, 27 \\
\hline I.L.M. &,- 13 &, 26 \\
\hline I.E. &,- 14 &, 23 \\
\hline I.N. &, 05 &, 65 \\
\hline I.C.K. &,- 17 &, 89 \\
\hline I.Inter. &, 12 &, 33 \\
\hline I.M. &,- 12 &, 33 \\
\hline I.Intra. &, 02 &, 84 \\
\hline $\begin{array}{l}\text { Leyenda. I.L.: Inteligencia Lingüística; I.L.M.: Inteligencia Lógico Matemática; I.E.: Inteligencia } \\
\text { Espacial; I.N.: Inteligencia Naturalista; I.C.K.: Inteligencia Corporal-Kinestésica; I.Inter: } \\
\text { Inteligencia Interpersonal; I.M.: Inteligencia Musical; I.Intra.: Inteligencia Intrapersonal. }\end{array}$ \\
\hline
\end{tabular}

\subsubsection{Correlaciones entre creatividad y rendimiento académico}

En este caso observamos se han obtenido correlaciones negativas y estadísticamente no significativas $(r=-, 15 ; p=, 20)$.

TABLA 7

Correlación entre creatividad y rendimiento académico

\begin{tabular}{|c|c|c|}
\hline & Rendimiento Académico & \\
\hline \multirow{3}{*}{ Creatividad } &,- 15 & Correlación de Pearson \\
\cline { 2 - 3 } &, 20 & Valor $\mathrm{p}$ \\
\cline { 2 - 3 } & 66 & $\mathrm{~N}$ \\
\hline
\end{tabular}

\section{DISCUSIÓN Y CONCLUSIONES}

El principal objetivo de este estudio fue analizar la relación existente entre el rendimiento académico, la creatividad y las inteligencias múltiples en una muestra de 66 alumnos de Educación Primaria. Los resultados muestran ausencia de correlación entre la creatividad y el rendimiento académico y entre la creatividad y las inteligencias múltiples. Observamos una correlación positiva y estadísticamente significativa entre las inteligencias múltiples y el rendimiento académico. Se pasa, a continuación, a realizar un análisis más detallado de los resultados estadísticos en base a las hipótesis planteadas y los estudios previos identificados en el marco teórico. 
JOSÉ JOAQUÍN CALDERA ORTIZ, FÁTIMA LLAMAS-SALGUERO Y VERÓNICA LÓPEZ-FERNÁNDEZ NEUROPSICOLOGÍA Y EDUCACIÓN: CREATIVIDAD, INTELIGENCIAS MÚLTIPLES Y RENDIMIENTO ACADÉMICO EN EDUCACIÓN PRIMARIA

De acuerdo con la primera hipótesis en la que se establecía la existencia de correlación entre el rendimiento académico y las inteligencias múltiples se ha constatado que se mantiene, existiendo correlaciones positivas altas entre el rendimiento académico y las inteligencias lingüísticas y lógico-matemáticas. Existen correlaciones positivas moderadas entre el rendimiento académico y las inteligencias naturalistas interpersonal e intrapersonal y musical, y correlaciones bajas entre el rendimiento académico y la inteligencia corporal-kinésica. Todas estas relaciones son estadísticamente significativas.

Estos resultados coinciden con investigaciones previas que indican que la inteligencia es una variable que correlaciona con el rendimiento académico (Andrade et al., 2000; Cascón, 2000; Machuca y Reyes, 2005; Pizarro y Clark, 2000). Cascón (2000) señalaba que la inteligencia era de los factores que mejor predicen el rendimiento académico recomendando el uso de test de inteligencia estandarizados como instrumento para detectar las posibles causas del fracaso escolar. En esa misma línea, Pizarro y Clark (2000) refirieron que las inteligencias múltiples se correlacionan de forma positiva entre ellas mismas y con el rendimiento académico. De forma más específica, Andrade et al., (2000) afirman que la inteligencia lingüística y la inteligencia lógico-matemática son las que mejor predicen el rendimiento académico. Este hecho se ratifica en el presente estudio puesto que son las dos inteligencias que muestran una correlación más alta.

En lo relativo a la segunda hipótesis, en la cual se planteaba la existencia de relaciones positivas y estadísticamente significativas entre la creatividad y las inteligencias múltiples, podemos afirmar que no se mantiene. Concretamente, se obtienen correlaciones negativas entre la creatividad y las inteligencias lingüística, lógico-matemática, espacial, corporal-kinésica y musical y correlaciones positivas entre la creatividad y el resto de inteligencias. En ambos casos son estadísticamente no significativas.

Estos resultados son similares a los encontrados en otros estudios (Rodríguez, Ezquerro, Llamas y López, 2016) que muestran correlación entre la creatividad y algunas de las inteligencias múltiples. Además, se han encontrado distintos trabajos en los que se constata de manera conceptual que la creatividad puede ser una forma distinta de inteligencia. Este hecho pone de manifiesto que al relacionar ambas variables se originan diferentes correlaciones estadísticamente significativas o no tanto de signo positivo como negativo (Getzels y Jackson, 1962; Mednick, 1962; Renzulli, 1977; Torrance, 1987; Wallach y Kogan, 1965).

En los estudios llevados a cabo por Torrance (1965), concluyen en que las diferencias entre la creatividad y el rendimiento académico pueden manifestarse de manera distinta y combinándose en múltiples formas. Esto se relaciona con los resultados obtenidos en la presente investigación. Debido al pequeño tamaño de la muestra, no se pueden sacar conclusiones fiables, pero teniendo en cuenta los resultados obtenidos se puede afirmar que no existe una relación directa entre los resultados académicos que obtienen los alumnos y los niveles de creatividad que poseen. 
JOSÉ JOAQUÍN CALDERA ORTIZ, FÁTIMA LLAMAS-SALGUERO Y VERÓNICA LÓPEZ-FERNÁNDEZ NEUROPSICOLOGÍA Y EDUCACIÓN: CREATIVIDAD, INTELIGENCIAS MÚLTIPLES Y RENDIMIENTO ACADÉMICO EN EDUCACIÓN PRIMARIA

La tercera hipótesis planteada señalaba la correlación positiva y estadísticamente significativa entre el rendimiento académico y la creatividad. No se confirma esta hipótesis puesto que se obtienen correlaciones negativas y no estadísticamente significativas.

De esta forma, obtenemos resultados similares a los encontrados por Garaigordobil y Torres (1997), donde creatividad y rendimiento académico apenas muestran factores correlacionales. Sin embargo, es conveniente destacar que existen otros estudios (Haddon y Litton, 1968; Karimi, 2000; Limiñana et al., 2010 y McCabe, 1991) que afirman que existe una pequeña relación entre ambas variables (rendimiento académico y creatividad). Además del número de la muestra, cabe destacar que desde el centro no se desarrollan actuaciones encaminadas a desarrollar el pensamiento creativo y el contexto que rodea a los alumnos también es muy poco favorecedor.

Una vez analizadas las hipótesis planteadas al inicio y contrastados los resultados con otras investigaciones se puede afirmar que la primera hipótesis que establecía correlaciones positivas y estadísticamente significativas entre el rendimiento académico y las inteligencias múltiples se mantiene. De acuerdo a estos resultados, se acepta la opción de trabajar las inteligencias múltiples como instrumento para mejorar los resultados académicos. Con la segunda y tercera hipótesis se pretendía comprobar la relación existente entre la creatividad y las inteligencias múltiples y la creatividad y el rendimiento académico respectivamente. En ambos casos se rechazan las hipótesis, mostrando los resultados la no existencia de relación entre las distintas variables. Esto ha podido suceder por la limitación de la muestra.

\subsection{Limitaciones y prospectiva}

Tomando como referente las conclusiones obtenidas en el presente estudio es posible establecer futuras líneas de investigación. Una primera posibilidad sería ampliar el tamaño de la muestra, llevándose a cabo en distintos centros educativos. Este aspecto permitiría tener un conocimiento más profundo de las distintas variables. Además, se podría llevar a cabo un estudio en dos momentos (pre y post) para conocer con exactitud cuál es la evolución del alumnado y la efectividad de los programas de intervención que se pudieran poner en marcha.

Como se señala en la introducción, es necesario establecer una relación más estrecha entre la neuropsicología y la educación (Marina, 2012). El conocimiento del funcionamiento cerebral es fundamental y obliga a replantear la base del sistema educativo. Se hace necesario establecer puentes que acerquen epistemológicamente y metodológicamente ambos campos (Benarós et al., 2010). Todo profesor debe conocer las bases cerebrales para poder entender como aprenden los alumnos y poder articular actuaciones educativas eficientes y eficaces.

Por último, cabe destacar que en la presente investigación se han encontrado una serie de limitaciones que es conveniente indicar para contextualizar los 
JOSÉ JOAQUÍN CALDERA ORTIZ, FÁTIMA LLAMAS-SALGUERO Y VERÓNICA LÓPEZ-FERNÁNDEZ NEUROPSICOLOGÍA Y EDUCACIÓN: CREATIVIDAD, INTELIGENCIAS MÚLTIPLES Y RENDIMIENTO ACADÉMICO EN EDUCACIÓN PRIMARIA

resultados y las conclusiones obtenidos en el mismo. Las limitaciones encontradas son las siguientes:

- El tamaño de la muestra, aunque es suficiente para llevar a cabo el estudio y realizar pruebas estadísticas paramétricas, no permite generalizar los resultados.

- Las variables medidas son complejas y multicausales. Estos aspectos dificultan establecer relaciones unívocas entre ellas.

- Las pruebas empleadas para medir las distintas variables objeto de estudio permiten hacerlo en un momento y en un contexto determinado, pero no son una medida objetiva y absoluta de las variables.

\section{REFERENCIAS BIBLIOGRÁFICAS}

Abalde, E.; Barca, A.; Muñoz, J. y Ziemer, M. (2009). Rendimiento académico y enfoques de aprendizaje: una aproximación a la realidad de la enseñanza superior brasileña en la Región Norte. Revista de Investigación Educativa, 27 (2), 303-319.

Andrade, M.; Miranda, C. y Freixas, I. (2000). Rendimiento académico y variables modificables en alumnos de 2do medio de Liceos Municipales de la comuna de Santiago. Revista de Psicología Educativa, 6 (2), 1-17.

Benítez, M.; Giménez, M. y Osicka, R. (2000). Las asignaturas pendientes y el rendimiento académico: ¿existe alguna relación? Descargado 21 de febrero de 2016. http//fai.unne. edu.ar/links/LAS \%2...20EL \%20RENDIMIENTO \%20ACADEMICO.htm.

Bernardo, J. y Caldero, J. F. (2000). Investigación cuantitativa. Madrid: RIALP.

Blasco, J. L.; Bueno, V.; Navarro, R. y Torregrosa, D. (2002). Educación emocional. Valencia: Generalitat Valenciana. Conselleria de Cultura i Educació.

Blázquez, A. (2009). Educación y creatividad. Revista Digital: Innovación y Experiencias Educativas, 14.

Campos, A. y González, M. A. (1993). Creatividad y rendimiento académico en estudiantes de bellas artes, ciencias y letras. Adaxe, 9, 19-28.

Cascón, I. (2000). Predictores del rendimiento académico en alumnos de primero y segundo de BUP. Descargado el 2 de marzo de 2016. http://campus.usal.es/ inico/investigacion/ jornadas/jornada2/comun/c19.html.

Corbalán, F.; Martínez, F.; Donolo, S.; Alonso, C.; Tejerina, M. y Limiñana, R. (2003). CREA. Inteligencia creativa. Una medida cognitiva de la creatividad. Manual. Madrid: TEA Ediciones.

Covington, M. V. (1984). The motive of self-worth. Motivation in education: student motivation, $1,77-113$.

Elshout, J. J. y Veenman, M. V. J. (1992). Relation between intellectual ability and working method as predictors of learning. Journal of Educational Research, 85 (3), 134-143.

Escalante, G. (2005). Creatividad y rendimiento académico. Centro de Investigaciones Psicológicas. Recuperado 22 de febrero de 2016. http://www.saber.ula.ve/bitstream/123456789/16176/1/creatividad-rendimiento.pdf.

Espíndola, J. L. (1996). Creatividad: Estrategias y Técnicas. España: Pearson. 
JOSÉ JOAQUÍN CALDERA ORTIZ, FÁTIMA LLAMAS-SALGUERO Y VERÓNICA LÓPEZ-FERNÁNDEZ NEUROPSICOLOGÍA Y EDUCACIÓN: CREATIVIDAD, INTELIGENCIAS MÚLTIPLES Y RENDIMIENTO ACADÉMICO EN EDUCACIÓN PRIMARIA

Fuentes, C.-R. y Torbay, A. (2004). Desarrollar la creatividad desde contextos educativos: un marco de reflexión sobre la mejora socio-personal. Revista Electrónica Iberoamericana sobre Calidad, Eficiencia y Cambio en Educación, 2 (1).

Garaigordobil, M. y Torres, E. (1996). Evaluación de la creatividad en sus correlatos con inteligencia y rendimiento académico. Revista de Psicología Universitas Tarraconensis, 18 (1), 87-98.

Gardner, H. (1983). Frames of mind: The theory of multiple intelligences. Nueva York: Basic Books.

Gardner, H. (1998). Mentes creativas: Una anatomía de la creatividad. Barcelona: Paidós.

Gardner, H. (2005). Inteligencias Múltiples. La teoría en la práctica. Barcelona: Paidós.

Gardner, H. (2006). Multiple intelligences: New horizons. Nueva York: Basic Books.

Getzels, J. W. y Jackson, P. W. (1962). Creativity and intelligence. Londres/Nueva York: John Wiley \& Sons, Inc.

Goleman, D. (1999). Inteligencia emocional. Barcelona: Kairós.

Guilford, J. P. (1967). The nature of human intelligence. Nueva York: Wiley.

Haddon, F. A. y Lytton, H. (1968). Teaching approach and the development of divergent thinking abilities in primary schools. British Journal of Educational Psychology, 38, 171-180.

Hernández, R. (2003). Metodología de la investigación. 3. a edición. México: McGraw-Hill.

Jensen, A. R. (1998). The g factor: The science of mental ability.

Jiménez, M. (2000). Competencia social: intervención preventiva en la escuela. Infancia y Sociedad, 24, 21-48.

Karimi, A. (2000). The relationship between anxiety, creativity, gender, academic achievement and social prestige among secondary school. Shiraz: University of Shiraz.

Lazear, D. (1991). Seven ways of teaching: The artistry of teaching with multiple intelligences. Palatine, IL: Skylight Publishing.

Limiñana, R. M.; Bordoy, M.; Juste, G. y Corbalán, J. (2010). Creativity, intelectual abilities and response styles: Implications for academic performance in the secondary school. Anales de Psicología, 26 (2), 212-219.

Machuca, L. y Reyes, A. (2005). Creatividad, inteligencia y aprendizajes escolares lingüísticos. Boletín de Investigación Educacional, Pontificia Universidad Católica de Chile, 20 (1), 95-104.

Marina, J. A. (2012). Neurociencia y Educación. Revista del Consejo Escolar del Estado, 1 (1), 7-13.

Martínez, A. (2007). La investigación en la práctica educativa: Guía metodológica de investigación para el diagnóstico y evaluación de los centros docentes. Madrid: Ministerio de Educación y Ciencia.

McCabe, M. P. (1991). Influence of Creativity ang Intelligence on academic performance. Journal of Creative Behavior, 25 (2), 116-122.

McKenzie, W. (1999). Multiple Intelligences Survey. Descargado el 11/4/2016. http://surfaquarium.com/MI/inventory.htm.

Mednick, S. (1962). The associative basis of the creative process. Psychological Review, 69, 220-232.

Moreno, J. A. (1992). La capacidad creadora y los aprendizajes escolares. Estudio de los factores constitutivos de la creatividad. Revista de Psicología de la Educación, 3 (9), 15-26.

Morgado, I. (2012). Claves neurocientíficas de la enseñanza y el aprendizaje. Revista del Consejo Escolar del Estado, 1 (1), 15-17. 
JOSÉ JOAQUÍN CALDERA ORTIZ, FÁTIMA LLAMAS-SALGUERO Y VERÓNICA LÓPEZ-FERNÁNDEZ NEUROPSICOLOGÍA Y EDUCACIÓN: CREATIVIDAD, INTELIGENCIAS MÚLTIPLES Y RENDIMIENTO ACADÉMICO EN EDUCACIÓN PRIMARIA

Navarro, R. E. (2003). El rendimiento académico: concepto, investigación y desarrollo. Revista Electrónica Iberoamericana sobre Calidad, Eficacia y Cambio en Educación, 1 (2), 1-15.

Neisser, U.; Boodoo, G.; Bouchard, T. J.; Boyking, A. W.; Brody, N.; Ceci, S. J.; Halperin, D. F.; Loehlen, J. C.; Perloff, R.; Sternberg, R. J. y Urbina, S. (1996). Intelligence: Knowns and unknowns. American Psychologist, 51, 77-101.

OECD (2012). Programme for International Students Assessment. Descargado 6 de marzo de 2016. http://educalab.es/documents/10180/19987/PISA-2012-technical-report-final. pdf/f954de11-ae51-4bdf-970c-6e6dc3f67adb.

OECD (2013). Programme for International Students Assessment. Descargado 6 de marzo de 2016. http://www.mecd.gob.es/dctm/inee/internacional/pisa2012/pisa2012lineavolumeni.pdf?documentId=0901e $72 \mathrm{~b} 81786310$.

OECD (2016). PISA 2015 Results (Volume I): Excellence and Equity in Education, PISA, OECD. Publishing, Paris. http://dx.doi.org/10.1787/9789264266490-en.

Pizarro, R. y Clark, S. (2000). Inteligencia múltiple lógico-matemática y aprendizajes escolares científicos. Revista de Psicología de la Universidad de Chile, 9 (11), 75-89.

Renzulli, J. (1977). The enrichment triad model. Mandsfield Center: Creative Learning Press. Smith, E. (2001). Implications of multiple intelligences theory for second language learning. Faculty of Education, 2 (1), 32-52.

Sternberg, R. J. (1985). Beyond IQ: A triarchic theory of human intelligence. Nueva York: Cambridge University Press.

Sternberg, R. J. (1988). A three-facet model of creativity. Cambridge: Cambridge University.

Torrance, E. P. (1965). Rewarding creative behavior. Englewood Cliffs.

Thurstone, L. L. (1939). Primary Mental Abilities. Chicago: University of Chicago Press.

Ullmann, G. (1972). Creatividad. Madrid: Rialp.

Wallach, M. A. y Kogan, N. (1965). Modes of thinking in children. Nueva York: Holt, Rinehart y Winston.

Weisberg, R. W. (1988). Problem solving and creativity. Nueva York: Cambridge University Press.

Weisberg, R. W. y Alba, J. W. (1981). An examination of the alleged role of «fixation» in the solution of several "Insight" problems. Journal of Experimental Psychology, 110 (2), 169-192. 